\title{
Design of a Compact and Versatile Bench Scale Tubular Reactor
}

\author{
R. Prasad 1 *, Gaurav Rattan ${ }^{2}$ \\ ${ }^{1}$ Department of Chemical Engineering \& Technology, \\ Institute of Technology, Banaras Hindu University, Varanasi 221005, U.P., India \\ ${ }^{2}$ Department of Chemical Engineering \& Technology, \\ Punjab University, Chandigarh 160014, India
}

Received: 10 February 2009, Accepted: 9 May 2009

\begin{abstract}
A compact and versatile laboratory tubular reactor has been designed and fabricated keeping in view of reducing capital cost and minimising energy consumption for gas/vapor-phase heterogeneous catalytic reactions. The reactor is consisted of two coaxial corning glass tubes with a helical coil of glass tube in between the coaxial tubes serving as vaporiser and pre-heater, the catalyst bed is in the inner tube. A schematic diagram of the reactor with detailed dimensions and working principles are described. The attractive feature of the reactor is that the vaporiser, pre-heater and fixed bed reactor are merged in a single compact unit. Thus, the unit minimises separate vaporiser and pre-heater, also avoids separate furnaces used for them and eliminate auxiliary instrumentation such as temperature controller etc. To demonstrate the system operation and illustrate the key features, catalyst screening data and the efficient collection of complete and accurate intrinsic kinetic data are provided for oxidation of $\mathrm{CO}$ over copper chromite catalyst. CO oxidation is an important reaction for auto-exhaust pollution control. The suitability of the versatile nature of the reactor has been ascertained for catalytic reactions where either volatile or vaporizable feeds can be introduced to the reaction zone, e.g. oxidation of iso-octane, reduction of nitric oxide, dehydrogenation of methanol, ethanol and iso-propanol, hydrogenation of nitrobenzene to aniline, etc. (C) 2009 CREC UNDIP. All rights reserved.
\end{abstract}

Keywords: Reactor, Bench scale tubular, Compact and versatile, Catalytic reactions, CO- oxidation

\section{Introduction}

Catalytic reactors are at the heart of the majority of chemical processes. Inside the catalytic reactor multiple chemical and physical processes take place at different length and time scales and often in different phases. The proper design of a catalytic reactor requires the knowledge of the rate of reaction and the selectivity as a function of the operating conditions. Preferably a rate expression based on the intrinsic reaction kinetics is used to translate laboratory data to pilot scale and further to an industrial unit. Without reliable kinetics, the reactor design is rather speculative and it is not really possible to evaluate the deviations and the dynamics that occur in the reactor. This constitutes a critical step in assessing the operational safety and environmental impact of the production unit (Berger et al., 2001). Catalytic fixed bed reactors are the main reactor type used for large-scale heterogeneously catalysed gas-phase reactions. They are also often the reactor of choice in the laboratory for kinetic studies.

* Corresponding Author, E-mail: rprasad.che@itbhu.ac.in, Tel: +91-9415268192, Fax +91-542-2368092 ) 
New ideas for bench scale reactors are needed to improve the efficient collection of complete and accurate intrinsic kinetic data. Better characterization of both laboratory scale and full-scale reactors are required. Development of new chemical reactors is dependent on advances in fundamental research and enabling technologies needed to apply this to reactor design. The following features have been identified as desired functionalities for the novel reactors (David and Sharon, 2001) of the future: (a) their capital cost has to be reduced, while heat and mass transfer characteristics have to be improved, (b) energy requirements have to be reduced, while safety standards should remain high (c) they should be multifunctional, in terms of chemical reactions and separation, capable of producing ultra pure products, (d) they should be versatile for carrying out various types of reactions and controllable, and (e) they should handle transient operation. System integration will lead to ultra low cost reactors.

The present work is an attempt to design a compact and versatile laboratory tubular reactor keeping in view of reducing capital cost, minimising energy consumption and the efficient collection of complete and accurate intrinsic kinetic data. The design is simple and easily constructed from glass by standard scientific glass blowing techniques. To demonstrate the system operation and illustrate the key features, catalyst screening data and the efficient collection of complete and accurate intrinsic kinetic data are provided in this paper for oxidation of $\mathrm{CO}$ over copper chromite catalyst. The suitability of the versatile nature of the reactor has been ascertained for catalytic reactions where either volatile or vaporizable feeds can be introduced to the reaction zone, e.g. oxidation of iso-octane, reduction of nitric oxide, dehydrogenation of methanol, ethanol and isopropanol, hydrogenation of nitrobenzene to aniline, etc.

\section{Materials and Methods \\ 2.1. Bench Scale Reactor Design and Fabri- cation}

The reactor was fabricated in the laboratory consisting of two coaxial corning glass tubes by standard scientific glass blowing techniques; detailed dimensions are shown in Figure 1. A helical coil of glass tube in between the coaxial tubes, serves as a vaporizer for liquid reactants and preheater of the vaporized reactants and any other gaseous reactant mixture. The descending part of the coil acts as a vaporizer for liquid flowing downward as a thin film. The vapour of the liquid and gaseous reactants get mixed well while flowing upward through ascending part of the coil and heated up to the reaction temperature before entering the catalyst bed kept in the mid of the inner tube as shown in the figure. There is a hole in the lower part of the outer tube to take care of breakage due to the expansion or contraction of air in between coaxial tubes as the unit is subjected to the variation of temperature from ambient to the reaction temperature.

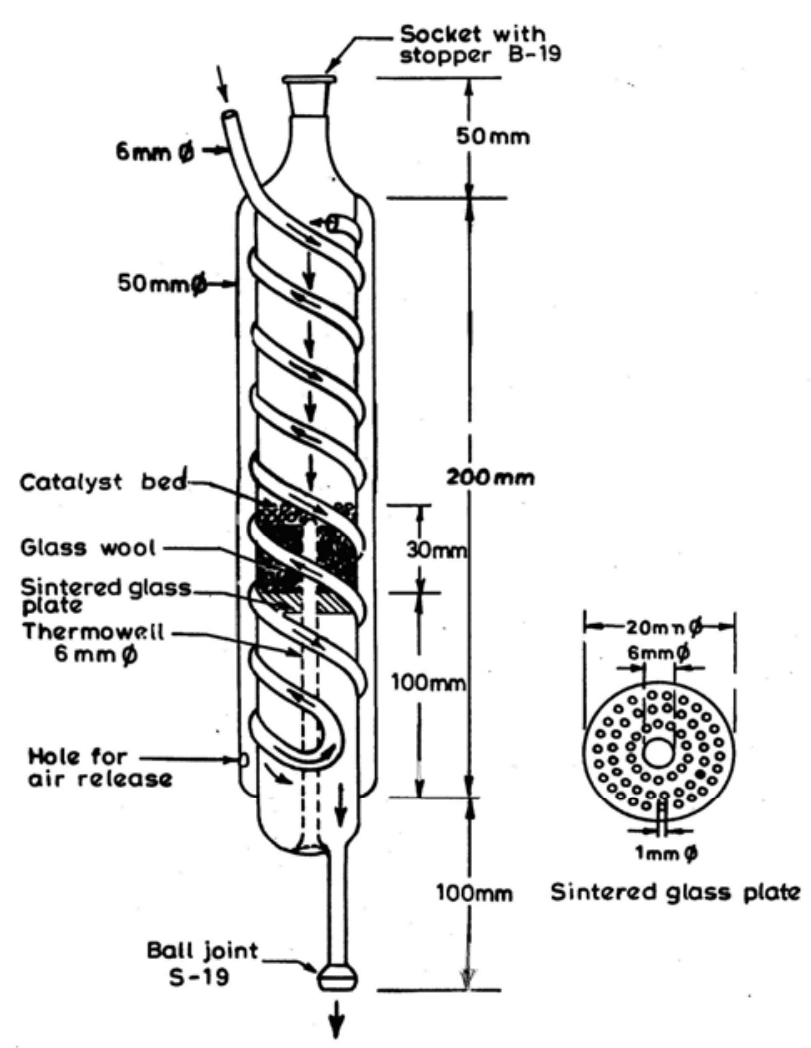

Figure 1. Schematic diagram of the reactor

In general bench scale tubular reactor consists of three units: (a) vaporiser (b) pre-heater and (c) fixed bed reactor, heated separately. Three separate heaters and their temperature controllers are needed to maintain the require temperatures. The attractive feature of the presently designed reactor is that the vaporizer, pre-heater, and the fixed bed reactor are merged together in a single unit. The compact unit not only minimizes separate vaporizer and pre-heater but also saves electrical energy by avoiding separate furnaces used for them. This reactor also eliminates auxiliary instrumentation such as temperature controller etc. Obviously fixed cost of single compact reactor, single heater and single temperature controller would be less than three separate units, their heaters and controllers. Also, it is natural that 
operating cost of energy consumption in a single heater would be minimum than three separate heaters. There is no such compact reactor has been reported in the open literature. This is our new contribution on the bench scale reactor.

The suitability of the versatile nature of the present reactor has been ascertained for catalytic reactions for gases as well as for vaporised liquids, and for gas and vaporised liquid reactants such as oxidation of carbon monoxide, oxidation of iso-octane, reduction of nitric oxide (auto-exhaust pollution control), and dehydrogenation of ethanol and iso-propanol, and hydrogenation of nitrobenzene to aniline, etc (important commercial reactions).

\subsection{Oxidation of CO Over Copper Chromite Catalyst}

The catalytic oxidation of carbon monoxide with the object of reducing air pollution is actually an important consideration when one thinks in terms of automobile emission control (Severino and Laine 1983). Copper chromite catalyst seems to be the most suitable for substituting noble metals employed as auto-emission control catalyst (Laine et al., 1990). The catalyst converts CO to harmless product $\mathrm{CO}_{2}$, found in the atmosphere which is useful to the plants.

$$
\mathrm{CO}+1 / 2 \mathrm{O}_{2} \text { (air) }=\mathrm{CO}_{2}
$$

Three copper chromite catalysts were prepared by calcining a complex precursor (ammoniac copper oxalate chromate) around 623 $\mathrm{K}$ in nitrogen (Cat-A), in air (Cat-B) and by prereducing Cat-B with CO (Cat-C). Preparation details are given elsewhere (Prasad, 2005). In situ pre-reduction of the catalyst (Cat-C) was carried out with $\mathrm{CO}$ / nitrogen mixture at $493 \mathrm{~K}$ for three hours. Composition of all the catalysts was identical having $\mathrm{Cu} / \mathrm{Cr}$ atomic ratio $1 / 1$. The only differences are the decomposition environments of the precursor and pre-reduction of the catalyst. The reactor was placed vertically in a split open microprocessor controlled furnace. The temperature control of the catalyst bed of $\pm 0.5 \mathrm{~K}$ was achieved. Following reaction conditions were maintained in the oxidation of CO: $100 \mathrm{mg}$ catalyst, particle size 100-150 mesh diluted to $10 \mathrm{ml}$ with alumina particles of the same size, Temperature $323-573 \mathrm{~K}, \mathrm{CO} /$ air ratio $1 / 40$, W/F $0-27$ g.h.mol-1 and pressure 1 atmosphere. Digital binary gas mixture (NUCON make) was used to feed CO/air mixture in required proportion to the reactor. Reactants and products were analysed for $\mathrm{CO}$ and $\mathrm{CO}_{2}$ with the help of an online gas chromatograph using porapack-Q column, methaniser and FID detector.

\section{Results and Discussion}

\subsection{Catalyst Screening}

The conversion of $\mathrm{CO}$ was calculated on the basis of values of $\mathrm{CO}$ concentration in the reactor

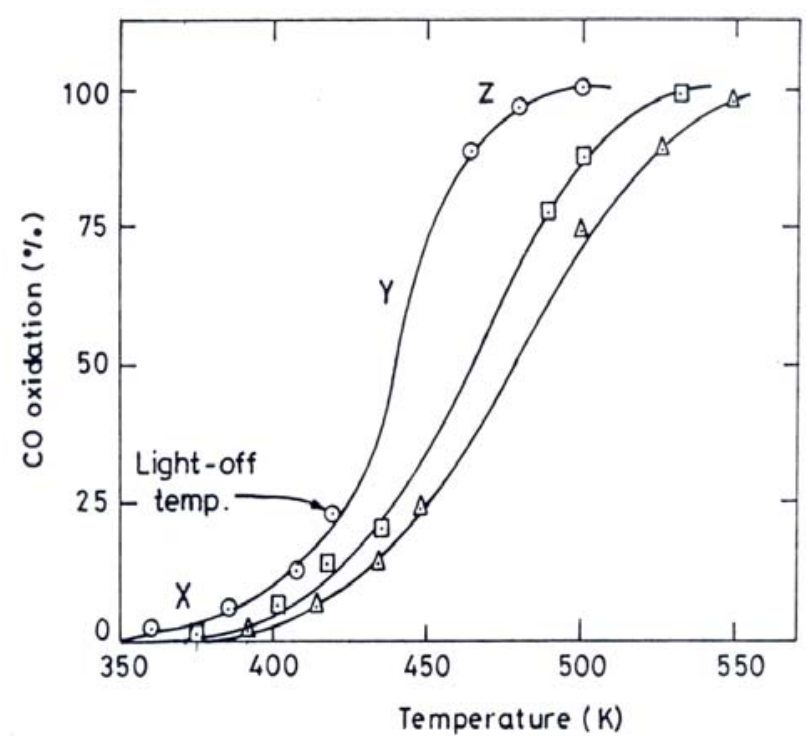

Figure 2. Effect of temperature on $\mathrm{CO}$ conversion, $\mathrm{CO} /$ air ratio $=1 / 40, \mathrm{~W} / \mathrm{F}_{\mathrm{CO}}=27 \mathrm{~g} \cdot \mathrm{h} \cdot \mathrm{mol}^{-1}$. $\circ$ Cat-A $\Delta$ Cat-B $\square$ Cat-C

input and output samples. The experimental results are shown in Figure 2.

All the catalysts show similar ignition behaviour. Three distinct zones can be visualized in Figure 2. In the first region-X below light-off temperature, rate is controlled by the kinetics of the chemical reaction. Catalyst performance in this region is dictated by the specific activity of the catalyst and by the dispersion of the metal. As the rate increases, heat builds up due to exothermic oxidation to the point where the catalyst lights off (region-Y). Eventually, the transfer of heat is limited and the system regains steady state (region$\mathrm{Z})$ at very high conversion. In this region mass transfer of gases to the catalysts is the rate determining step. Figure 2 shows that the conversion of $\mathrm{CO}$ for cat- $\mathrm{C}$ is higher than that for cat-B and it is highest for cat-A at all temperatures.

The durability of the catalysts was tested by carrying out the reaction for 50 hours at $500 \mathrm{~K}$. The results are plotted in Figure 3. In the beginning, exothermic effect of the reaction increased the bed temperature above the pre-adjusted value of $500 \mathrm{~K}$. After the temperature was stabilized, a progressive deactivation of the catalysts was observed. It is clear from Figure 3 that the activities 


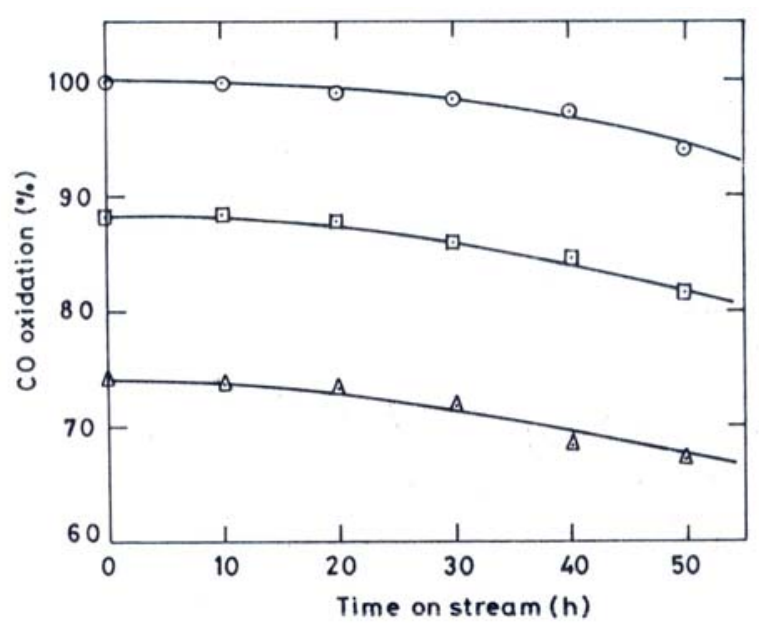

Figure 3. Effect of catalyst ageing on $\mathrm{CO}$ oxidation: Temp. $500 \mathrm{~K}$; CO/air ratio $1 / 40 ; \mathrm{W} / \mathrm{F}_{\mathrm{CO}}=$ 27g.h.mol-1. o Cat-A $\Delta$ Cat-B $\square$ Cat-C

of all the catalysts dropped down by about $6-7 \%$ during 50 hours of operation. The conversion of CO by Cat-A was maintained about $94 \%$ even after 50 hours of continuous use.

\subsection{Kinetics of $\mathrm{CO}$ oxidation}

The kinetics of the oxidation of $\mathrm{CO}$ over the best screened cat-A were studied in the reactor using a lean mixture of CO in air (1/40), particle size 100-150 mesh and

$\mathrm{W} / \mathrm{F}_{\mathrm{CO}}$ values 0 - 27 gcat.h.mol-1. The mass of the catalyst taken was $0.1 \mathrm{~g}$ diluted to $10 \mathrm{ml}$ with alumina of the same size. Meaningful kinetic data can be obtained from a packed bed reactor only if the flow pattern within the reactor resembles plug flow. In present study reactor satisfied the criteria of plug flow behaviour. The reactor was operated essentially as a differential reactor with low conversion of $\mathrm{CO}$ below $10 \%$. The external and internal mass transfer effects were absent within the experimental region of kinetics measurements, these were checked experimentally. The precautions taken to avoid a temperature rise of the catalyst bed were sufficient to assure isothermal behaviour. The kinetics treatment follows the empirical rate law equations as favoured by Levenspiel (1999). The experimental data at four different temperatures are presented in Figure 4 . The rate of reaction at any conversion was obtained by taking slope of $\mathrm{CO}$ conversion ( $\left.\mathrm{X}_{\mathrm{CO}}\right)$ versus $\mathrm{W} / \mathrm{F}_{\mathrm{CO}}$ plot. Once the rate of reaction is known as a function of conversion or concentra-

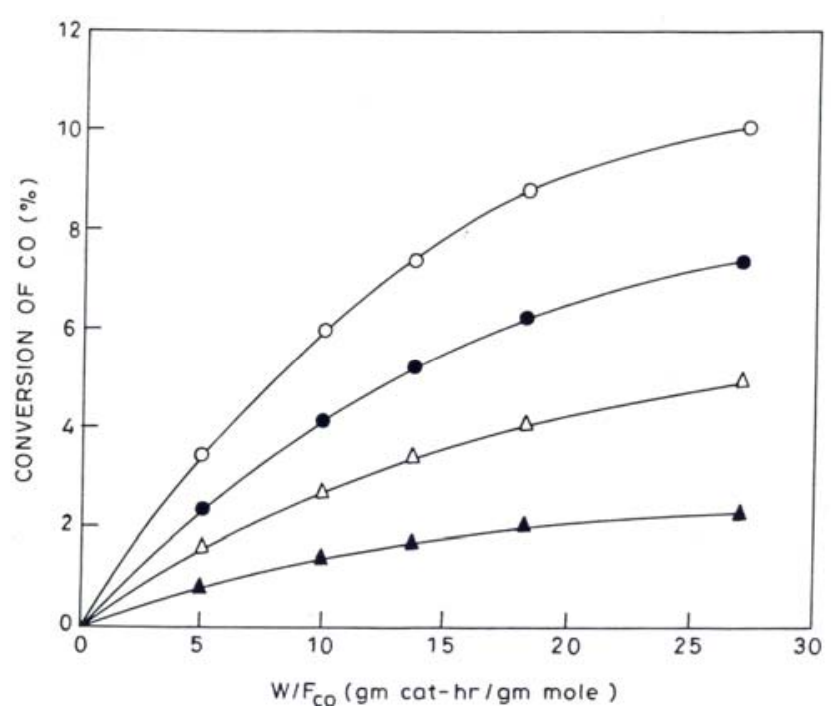

Figure 4. Plot of conversion of $\mathrm{CO}$ vs. W/F $\mathrm{FO}$ Temp: (०) $400 \mathrm{~K}, \quad$ (•) $390 \mathrm{~K}, \quad$ ( $\Delta) 380 \mathrm{~K}$, $370 \mathrm{~K}$

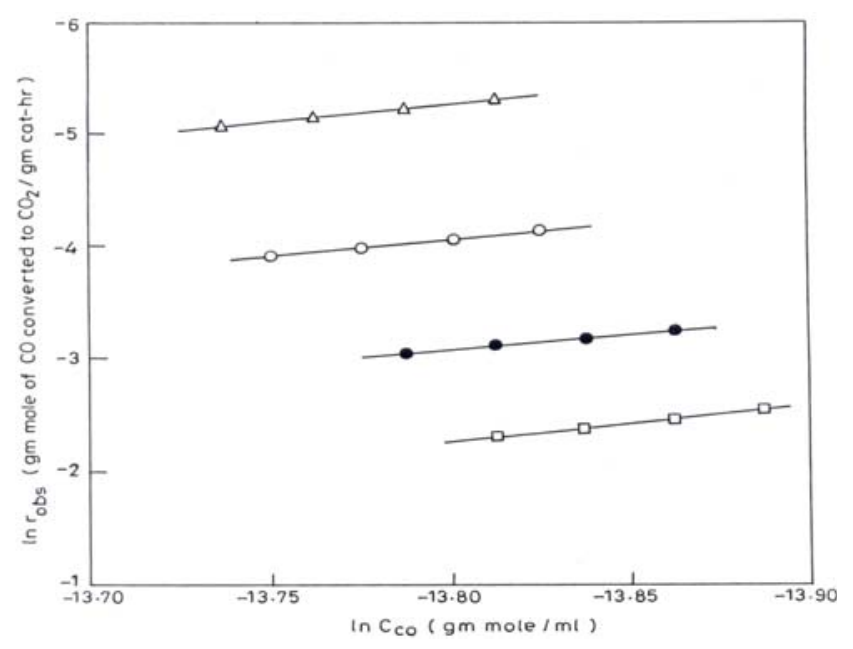

Figure 5. Plot of $\ln \left(\mathrm{r}_{\mathrm{obs}}\right)$ vs. $\ln \left(\mathrm{C}_{\mathrm{CO}}\right)$

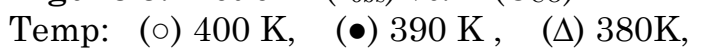

$(\Delta)$ $370 \mathrm{~K}$

tion of reactant, an empirical power law type equation can be fitted:

$$
r_{o b s}=k^{\prime}\left(C_{O 2}\right)^{m}\left(C_{C O}\right)^{n}
$$

In the present study a lean mixture of reactant, $2.5 \% \mathrm{CO}$ in air was used, therefore oxygen was in large excess and the rate expression reduces to the pseudo $\mathrm{n}^{\text {th }}$ order:

$$
\begin{aligned}
r_{o b s} & =k\left(C_{C O}\right)^{n} \\
C_{C O} & =\left(C_{C O}\right)_{\text {inlet }}\left(1-X_{C O}\right)
\end{aligned}
$$


The rate constant $\mathrm{k}$ is a function of temperature and can be expressed by Arrhenius equation:

$$
k=A \exp (-E / R T)
$$

Linear plots were obtained when $\ln \left(\mathrm{r}_{\mathrm{obs}}\right)$ vs. $\ln \left(\mathrm{C}_{\mathrm{CO}}\right)$ were plotted (Figure 5). Slopes and intercepts of the lines gave order of reaction and $\ln (\mathrm{k})$ respectively. Order of reaction was found to be 0.7. The reaction rate constants follow Arrhenius law. The activation energy and frequency factor (A) were calculated measuring the slope and intercept respectively of the plot $\ln (\mathrm{k})$ vs. $1 / \mathrm{T}$ as shown in Figure 6.

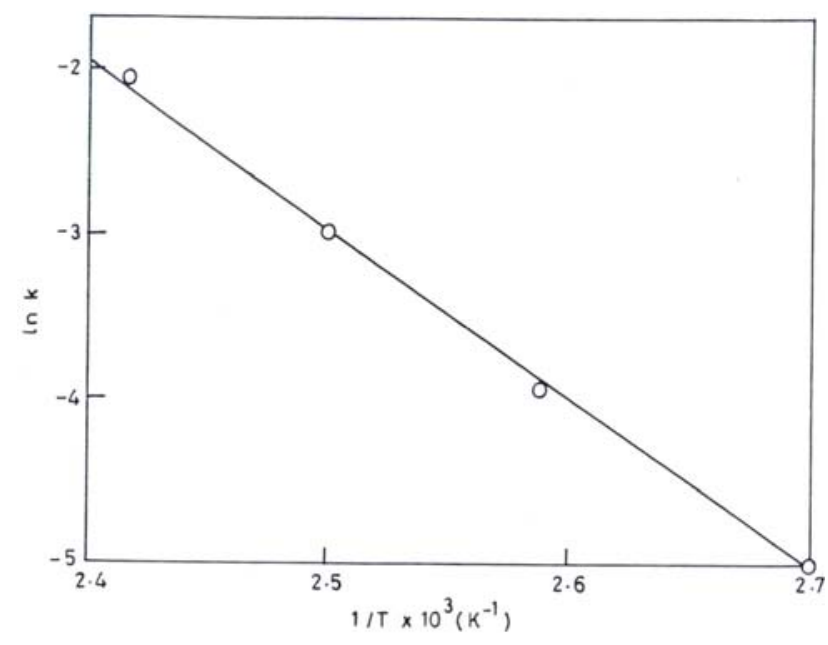

Figure 6. Arrhenius plot

On the basis of the experimental findings the rate of $\mathrm{CO}$ oxidation in the temperature range of $370-400 \mathrm{~K}$ on the copper chromite catalyst can be expressed as:

Rate $=2.02 \times 10^{4} \exp (-12290 / R T)\left(C_{C O}\right)^{0.7}$

The above empirical rate expression can be adequately used for the design of $\mathrm{CO}$ converter.

Similarly, the suitability of the versatile nature of the present reactor has been ascertained for catalytic reactions for oxidation of iso-octane, reduction of nitric oxide (auto-exhaust pollution control), and dehydrogenation of ethanol and isopropanol, and hydrogenation of nitrobenzene to aniline, etc (important commercial reactions).

\section{Conclusion}

A low cost, compact and versatile laboratory tubular reactor for vapour phase heterogeneous catalytic reactions has been designed and fabricated. The vaporiser, pre-heater and fixed bed reactor are merged in a single unit. Thus, not only the fixed cost of the reactor system is decreased but also the operating cost of energy consumption is minimised. The suitability of the reactor has been demonstrated for screening of the catalysts, performance measurements and the efficient collection of complete and accurate kinetic data for oxidation of CO over copper chromite catalyst. Experience in using the device on a variety of industrial catalysis programs has shown that it is a versatile and ideal device that can handle any gas-phase reaction where either volatile or vaporizable feeds can be introduced to the reaction zone.

\section{Acknowledgment}

The authors are gratefully acknowledging the financial support given for the project by the Department of Science and Technology, India.

\section{References}

[1] Berger, R. J.; Stitt, E. H.; Marin, G. B.; Kaptejn, F.; and Moulijn, J. A. 2001. Chemical Reaction Kinetics in Practice. Cattech. 5, 30-60.

[2] David, H. Klipstein, and Sharon Robinson 2001. Citing Intertnet sources URL http:// www.Chemicalvision 2020.org/pdfs/ reaction_roadmap.pdf. Vision 2020: Reaction Engineering Roadmap 9-24.

[3] Laine, J.; Brito, J.; and Severino, F. 1990. Surface Copper Enrichment by Reduction of Copper-Chromite Catalyst for Carbon Monoxide Oxidation. Catal. Letters 5: 45-54.

[4] Levenspiel, O. 1999. Chemical Reaction Engineering, John Wiley Eastern \& Sons, New York, 380.

[5] Prasad, R. 2005. Highly Active Copper Chromite Catalyst Produced by Thermal Decomposition of Ammoniac Copper Oxalate Chromate. Materials Letters 59: 3945-3949.

[6] Severino, F., and Laine, J. 1983. Effect of Composition and Pre-treatments on the Activity of Copper-Chromite-based Catalysts for Oxidation of Carbon Monoxide. Ind. Eng. Chem. Prod. Res. Dev. 22: 396-401. 